Aus dem Institut zur Erforschung der Infektionskrankheiten in Bern.

\title{
Aphoristische Betrachtungen über einige praktisch und theoretisch wichtige Punkte der Desinfektionslehre.
}

\author{
Von Prof. Dr. W. Kolle.
}

Wenn wir die Lehre von der Desinfektion überblicken, so finden wir viele Fragen so gut wie abgeschlossen. Das gilt in erster Linie für die Bewertung der Desinfektionskraft in vitro der chemischen, in Wasser löslichen Desinfektionsmittel. Es ist das große und unvergängliche Verdienst von Robert Koch, daf er die Desinfektionsmittellehre durch seine Entdeckungen auf eine sichere Basis stellte; namentlich dadurch, dab el zeigte, wie man die Bakterien, bzw. ihre Sporen zur Prüfung von Desinfektionsmitteln benutzen kann. Seit den grundlegenden Forschungen von Robert $\mathrm{Koch}$ und von seinen ersten Mitarbeitern Gaffky, Loeffler und Proskauer über die Methodik der Prüfung von flüssigen Desinfektionsmitteln, sind in zahlreichen Arbeiten mit Hilfe dieser Methodik die geeignetsten Mittel zur Zerstörung der verschiedensten Infektionsstoffe, wie sie außerhalb des Menschen- und Tierkörpers an Gebrauchsgegenständen, z. B. an Wäsche, Betten, Kleidern, sowie dem Trinkgeschirr, in den Ex- und Sekreten des Menschen vorkommen, bestimmt. Es herrschen nur noch geringfügige Differenzen in den Auffassungen, welche von den zahlreichen geeignet gefundenen Chemikalien für den einzelnen Fall herangezogen werden sollen. Zum Teil hängt es von dem Geschmack oder von äußeren Umständen, zu denen namentlich der Preis der Desinfektionsmittel gehört, ab, ob im einzelnen Falle der Karbolsäure und den Kresolen, dem Sublimat, dem Lysol oder der Schwefelsäure, Salzsäure, Karbolschwefelsäure oder der Kalkmilch, dem Aetzkalk etc. der Vorzug gegeben werden soll. Auch auf theoretischem Gebiete sind namentlich dank den Arbeiten von Paul und Krönig Fortschritte bezüglich der Erklärung einzelner Vorgänge, bzw. des inneren Zusammenhanges der Prozesse, die sich bei der Desinfektion abspielen,

1) Bei einem Tier wurde außer der spezifischen Keratitis noch eine spezifische Iritis mit Knötchenbildung beobachtet.

2) E. H of $\mathrm{fman} \mathrm{n}$, Berliner klinische Wochenschrlft 1907 , S. 351, Dermatologische Zeitschrift, August 1906, S. 563. - 3) G reeff und Clause n, Spirochaeta pallida bei experimentell erzeugter, interstitieller Hornhautentzlindung. Deutsche medizinische Wochenschrift 1906 , No. 36 . - 4) Tom a s z e w s ki, Uebertragung der experimentellen Augensyphilis des Kaninchens von Tier zu Tier. Munchener medizinische Wochen. schrift 1907 , No. 21, S. 1023 .

5) Anmerkung bei der Korrektur: In einem weiteren Präventivversuch sind von sechs Kaninchen die vier mit Atoxyl behandelten gesund geblieben, während die beiden mnbehandelten Kontrolltiere erkrankt sind. erzielt worden, Krönig und Paul haben die Ionenlehre mit Erfolg zur Erklärung komplizierter Vorgänge auf diesem Gebiet herangezogen.

Ein Beispiel möge dies erläutern. Man nahm früher an, dab bei der Verwendung von Sublimat der Gehalt der Lösung an Quecksilberchlorid das Entscheidende für die Größe der Desinfektionskraft dieses Präparates sei. Auf Grund der Ionentheorie mub man die Verhältnisse aber anders beurteilen. Man muß nach dieser Theorie annehmen, daß sich alle gelösten Salze im Zustande einer mehr oder weniger rasch verlaufenden Spaltung befinden und dab hierbei die sogenannten Ionen, d. i. elektropositive und elektronegative Teile, die mit großer chemischer Affinität behaftet sind, abgestoßen werden. Je größer diese Dissoziation, desto stärker ist die desinfizierende Kraft einer Lösung und umgekehrt. Ferner ist von Wichtigkeit, welche Art von Ionen bei der Dissoziation entstehen. Bei Sublimat z. B. müssen Quecksilberionen entstehen, wenn überhaupt eine Desinfektionswirkung eintreten soll. Aber die Verhältnisse liegen hier sehr kompliziert, denn auch die Art der auber den Quecksilberionen in der Lösung entstehenden Ionen, sowie die nicht dissoziierten Salze spielen eine Rolle bei der Desinfektionswirkung.

So wird durch diese Untersuchungen verständlich, warum manche Zusätze zu den desinfizierenden Lösungen störend auf den Desinfektionsvorgang einwirken können, andere fördernd. Auch dann, wenn diese Zusätze keine direkten Verbindungen mit den Desinfektionsmitteln eingehen, kann es doch bei der Lösung infolge der Gegenwart anderer Körper zu weitgehenden Veränderungen in bezug auf die chemisch-physikalischen Bedingungen der Mischungen kommen. Für die Praxis ergeben sich aus der Heranziehung der lonenlehre für die Desinfektionsversuche, wie sie von Krönig und Paul mit Erfolg unternommen worden ist, drei Folgerungen: einmal, möglichst chemisch reine Präparate zu benutzen; zweitens ist es notwendig, die Vorschriften für die Lösung und Anwendung der Desinfektionsmittel genau zu befolgen; drittens aber muß die Kombination verschiedener

6) Uhlenhuth, Oross und B|ckel, Untersuchungen über die Wirkung des Atoxyls auf Ting A toxyle 4. 
Chemikalien ins Auge gefaßit werden. Durch Verwendung z. B. von zwei Präparaten in proportional kleineren Mengen läbt sich, wie theoretisch zu erwarten, unter Umständen ein viel größerel' Desinfektionserfolg erzielen, als durch Verwendung eines Mittels in proportional viel höherer Konzentration der Lösung, als die beiden kombinierten sie besitzen.

Nicht weniger geklärt sind die Anschauungen über die Anwendungsweise und Leistungsfähigkeit der Dampfdesinfektion. Auch hierfür sind Kochs und seiner Mitarbeiter Ver. suche grundlegend geworden. Durch die Mitarbeit der Technik, die sich die Konstruktion tadelloser Apparate, sowohl stabiler wie transportabler, oder mit strömendem wie gespanntem Dampf arbeitender hat angelegen sein lassen, ist es heutzutage dahin gekommen, daß die Dampfdesinfektion überall, auch zum Teil auf dem Lande, möglich ist, und zwar in einer den Erfolg stets garantierenden Weise. Es hängt von der Größe des Betriebes oder äußeren Umständen, bei denen auch wieder die Preisfrage eine Rolle spielt, ab, welche Konstruktion des Apparates gewählt wird und ob man mit strömendem oder gespanntem Dampf arbeitet.

Daß es sich bei den berührten Punkten zum großen Teil, soweit sie die Praxis der Desinfektion betreffen, um abgeschlossene Probleme handelt, ist so recht ersichtlich aus den Ausführungsbestimmungen zum neuen preuBischen Seuchengesetz vom Jahre 1905. Die Leitgedanken dieses in der Medizinalabteilung des preuBischen Kultusministeriuns unter Zuziehung von zahlreichen Fachgelehrten zustande gekommenen Gesetzes sind in einer den Mitgliedern des in Berlin tagenden Kongresses fül Hygiene und Demographie zugänglich gemachten Denkschrift von M. Kirchner behandelt worden, der sich um das Zustandekommen und die Ausarbeitung des Gesetzes große Verdienste erworben hat.

Viel mehr der experimentellen Bearbeitung bedürftig ist die so wichtige Frage, wie sich die Infektionsstoffe im lebenden Körper des Menschen und der Tiere und an ihrer Oberfläche abtöten lassen. Bezüglich der Abtötung von Infektionserregern mittels chemischer Präparate sind neuerdings sehr wichtige Ergebnisse erzielt worden bei Protozoenkrankheiten der Menschen und Tiere. Es ist gelungen, die Trypanosomen der experimentellen Tsetsekrankheit durch Farbstoffe (Trypanrot Ehrlichs, Brillantgrün Wendelstadts) sowie Arsenpräparate (empfohlen von Laveran, neuerdings von F. Loeffler) innerhalb des Tierkörpers abzutöten und das Tier so zu heilen. Auch die Trypanosomen der Schlafkrankheit und einige Spirochätenarten lassen sich durch Atoxyl (Koch, Ayres, Kopke u. a.) im lebenden Körper vernichten. Bei den Bakterienkrankheiten ist die Desinfektion der Gewebe des infizierten Körpers mit Hilfe von chemischen Präparaten noch nicht gelungen. Die bakteriziden Sera sind bis jetzt die einzigen Stoffe, mittels deren eine „innere Desinfektion“ gelingt. Es muß als eines der medizinisch wichtigsten Probleme der Chemie bezeichnet werden, zur Auffindung von Mitteln zu gelangen, die für den Körper des Tieres und des Menschen ungiftig oder wenig giftig sind, für die Bakterien aber auch bei Gegenwart von EiweiB in geringsten Mengen abtötend wirken, sodaß sie auch in den Körperflüssigkeiten die bakteriellen Krankheitserreger, namentlich bei chronischen Infektionen, direkt vernichten. Dieser Weg wird von Bechhold in Ehrlichs Institut nouerdings beschritten.

Bei weitem aussichtsreicher ist die Desinfektion der Körperoberflächen, wie sie zur Entkeimung der Haut des Patienten und zur Reinigung der Hände des Arztes bei chirurgischen Operationen eine grobe Rolle spielt. Da die Frage der Händedesinfektion neuerdıngs wieder erörter't wird und zur Auffindung neuer Verfahren geführt hat, um keimarme oder keimfreie Hände zu erhalten, soll in dem Teil II kurz auf sie eingegangen werden.

$$
1 .
$$

In der Entwicklung begriffen muß unbedingt das Problem der Wohnungsdesinfektion betrachtet werden; ja es bestehen noch erhebliche Meinungsdifferenzen bei den Hygienikern über die Wohnungsdesinfektion im allgemeinen, und namentlich über die Ausführung der sogenannten Schlubdesinfektion. Die Schlußdesinfoktion ist auch in neuen preufischen Seuchen- gesetze abgegrenzt und gegenübergestellt der Desinfektion während der Dauer der Krankheit.

Es ist namentlich Robert Koch zu verdanken, daß man auf die Desinfektion während der Dauer der Krankheit den gröbten Wert legt. Durch die sinngemäße Ausführung der Desinfektion aller Sekrete und Exkrete des Kranken wird eine Verseuchung der Wohnung verhütet. Wenn eine Desinfektion aller infektiösen Stoffe, die ein Kranker liefert, frühzeitig vom Arzt, nämlich mit dem Momente, wo die Behandlung beginnt, angeordnet wird und wenn hierbei auf Grund der epidemiologischen Beobachtung von den Aerzten nicht schematisierend, sondern individualisierend vol'gegangen wird, so kann eine Infektion des Zimmers, wenn auch nicht verhütet, so doch sehr beschränkt werden. Die Desinfektion während der Dauer der Krankheit schließt ferner in sich, dab alles, was die Wohnung infizieren kann, nicht aus dem Krankenzimmer herauskommt, ohne desinfiziert zu sein; namentlich EB- und Trinkgeschirre und Gefäße, infizierte Leib- und Bettwäsche, Verbandstoffe, müssen im Krankenzimmer selbst durch die geeigneten Maßnahmen desinfiziert werden. Auch das Pflegepersonal muß den Körper, namentlich die Hände, sowie die besonders für diese Zwecke geeignete Kleidung nach näherer Anweisung des Arztes regelmäßig desinfizieren.

Die SchluBdesinfektion dagegen bezweckt, alle diejenigen Infektionsstoffe, welche dieser fortlaufenden Desinfektion entgangen sind, zu zerstören. Es handelt sich hier hauptsächlich um die beim Sprechen, Niesen oder Husten in Form von Tröpfchen, namentlich im Staube abgelagerten infektiösen Sekretteilchen. Sie hat sich nach dem neuen preubischen Seuchengesetz auf alle von dem Kranken benutzten Räume und Gegenstände zu erstrecken, bei welchell anzunehmen ist, daß sie mit dem Krankheitserreger behaftet sind, und soweit ihre Desinfektion nicht schon während der Krankheit erfolgt ist. Es ist ein großes Verdienst des Breslauer Hygienikers Flügge, die ganze Prage der Wohnungsdesinfektion experimentell geklärt und in gute Bahnen gelenkt zu haben. Wir wissen jetzt, daß sich mit e in em einzigen Verfahren oder e i $n$ e $m$ Desinfektionsmittel allein eine infizierte Wohnung und die in ihr enthaltenen Gegenstände, Möbel, Vorhänge etc. nicht von den Krankheitserregern befreien lassen. Es ist vielmehr notwendig, neben den chemischen, flüssigen Desinfektionsmitteln, sowie neben den mechanischen Faktoren der Reinigung und Säuberung auch gasförmige Desinfektionsmittel heranzuziehen. Von den letzteren kann auf Grund der experimentellen Prüfungen heutzutage wohl nur noch das Formaldehyd in Frage kommen. Das Schwefeldioxyd besitzt zwar in den hohen Konzentrationen, wie sie neuerdings mit Hilfe des Claytonapparates erzielt werden, neben seinen insektenfeindlichen Eigenschaften gute Desinfektionseffekte, ist aber wegen seiner die Möbel etc. beschädigenden Wirkungen für die Wohnungsdesinfektion nicht brauchbar. Wir kennen bis jetzt kein anderes Mittel, welches auf die Gebrauchsgegenstände der Wohnung, Möbel, Stoffe, Tapeten etc. so wenig schädigend einwirkte wie das Formaldehyd und eine gleich große Desinfektionswirkung besäße. Es ist das Verdienst von Flügge und Rubner, nachgewiesen zu haben, daß eine Vorbedingung für die Wirkung des gas. förmigen Formaldehyds die Gegenwart von viel Wasserdampf im Raum ist; die Luft muß vollständig oder annähernd gesättigt sein mit Wasserdämpfen, welche sich später niederschlagen und dabei mehr oder weniger große Mengen von Formaldehyd aufnehmen. Es ist also in letzter Instanz die Desinfektionskraft des Formaldehydgases eine solche des Formaldehyds in flüssiger Form. Zur Verdampfung des Formalins sind Apparate konstruiert worden, deren Aufgabe es ist, nicht nur genügende Mengen gasförmigen Aldehyds, sondern auch von Wasserdampf zu liefern. Es sind eine große Anzahl solcher Apparate empfohlen worden, von denen in Deutschland die bekanntesten diejenigen von Flügge, Lingner, Czaplewski, Schering, Proskauer und Elsner geworden sind. Die Apparate haben sich vielfach eingebürgert und es sind deren viele Tausende in Deutschland in Gebrauch! So sehr man sich über die weite Verbreitung del' Formaldehyddesinfektion freuen kann, so kann man sich doch nicht darüber täuschen, dal die Gewinnung gasförmigen Formal. dehyds durch Verdampfung mittels dieser Apparate keineswegs 
ein Idealverfahren darstellt. Denn die Apparate erfordern nicht unerhebliche Anschaffungs- und Reparaturkosten. Man bedarf eines Wagens oder Dreirades bei ihrem Transport, was beim Desinfizieren auf dem Lande unter Umständen nicht leicht zu bewerkstelligen ist. Uazu kommt die Feuergefährlichkeit der Apparate, die im Raum oder im Hause, in dem sich feuergefährliche Gegenstände befinden, aufgestellt werden müssen. Zur Desinfektion ganz großer Räume, z. B. von Hallen, Krankensälen, Bahnhof́swartesälen etc. sind außerdem mehrere Apparate notwendig, die selbst in größeren Städten oft nicht zur Verfügung stehen. Es liegt im Interesse einer zielbewußten Bekämpfung der Infektionskrankheiten, zu deren Durchführung auch die Wohnungsdesinfektion gehört, daß die Formaldehydverfahren möglichst vereinfacht werden.

Diesem Streben nach Vereinfachung ist das sogenannte A utanverfahren entsprungen.

Die von Eichengrïn aufgefundene Methode beruht auf dem Prinzip der Entwicklung des Formaldehyds in statu nascendi durch chemische Umsetzung. Eichengrün baute sein Vertahren auf der Tatsache auf, daß Metallsuperoxyde, z. B. Baryum- oder StrontiumSuperoxyd, mit Paraform gemischt, in trockenem Zustande unwirksam sind, bei Hinzufügung von Wasser aber sehr energische chenische Umsetzungen eingehen. Bei Gegenwart von Wasser fïhrt das Paraform nämlich eine Katalyse der Superoxyde herbei. Dadurch wird Alkalihydroxyd frei und entpolymerisiert in statu nascendi das Paraform. Bei dieser chemischen, sehr rasch verlaufenden Umsetzung wird in wenigen Augenblicken eine große Menge Wärme frei, welche das hinzugefügte Wasser in kurzer Zeit zur Verdampfnng bringt. Es werden also Formaldehyd und Wasserdampf gleichzeitig durch chemische Umsetzungen gewonnen. Ein Teil des Paraforms wird hierbei allerdings nicht zu Formaldehyd entpolymerisiert, sondern zu Ameisensäure umgewandelt. Das trockene Präparat Autan ist eine Misching von 29 Teilen Paraform mit 71 Teilen Baryumsuperoxyd. Wenn man aus diesem Pulver das Formaldehyd entwickeln will, so bringt man es in einen Waschzuber und versetzt es mit einer ganz bestimmten Menge Wasser, nämlich $80 \%$ des Autangewichtes. Auf die genaue Abmessung des Wassers kommt viel an, denn von der Wassermenge ist die Wärmeentwicklung und damit äie Intensität der Wasserverdampfung und der Formaldehydentwicklung abhängig. Wenn zu viel Wasser vorhanden ist, wird sich ein Teil des Formaldehyds in dem überschüssigen Wasser lösen und eine wässrige Formaldehydlösung (Formalin) bilden, anstatt zu verdampfen. Bei Anwendung einer zu geringen Menge Wassers wird nicht genug Superoxyd zerlegt, sodaß also die zur Entpolymerisierung des Paraforms nötige Menge freien Alkalihydroxyds fehlt. Ich habe mit den mir von den Elberfelder Farbwerken gütigst zır Verfügung gestellten Autanimengen selbst, sowie in Gemeinschaft mit den Herren Heller und Tomarkin ${ }^{1}$ ) eine große Anzahl von Versuchen in den verschiedensten Räumlichkeiten, größeren und kleineren, in Straßenbahn- und Eisenbahnwagen, bei den verschiedensten Temperaturen und unter Benutzung der verschiedensten Mengen des Autans angestellt. Die Erfolge der Desinfektion wurden durch Auslegen von Proben stets kontrolliert. Die Proben, bestehend aus verschiedenen Bakterienkulturen, Staphylococcen, Typhusbazillen, Milzbrandsporen, Bacterium coli, wurden teils an Seidenfäden angetrocknet, teils in feuchtem Zustande an den verschiedensten Teilen des Raumes ausgelegt. Es wurden die Objekte nachher, teils mit Ammoniak-, teils ohne Ammoniakspülung auf feste und flüssige Nührböden tabertragen und mehrere Tage lang bebrütet.

Die bis in die letzte Zeit von uns fortgesetzten Versuche haben nun ergeben, daß das Autanverfahren ein durchaus zuverlässiges ist. Allerdings genügen die ursprünglich von der Fabrik in Aussicht genommenen Mengen nicht. Bei ungünstigen Verhältnissen, namentlich dann, wenn durch die natürliche Ventilation ein großer Gasverlust eintritt, oder wenn sich im Raume einzelne kühle Flächen finden, an denen die Niederschlagsbildung besonders stark ist, reicht der Formaldehydgehalt der Luft bei Benutzung der von der Fabrik vorgeschriebenen Mengen des Autans nicht aus. Es ist dann notwendig, $50 \%$, mindestens aber $35 \%$ mehr zu nehmen, als die Fabrik es verlangt. Da die Umsetzung des Autans so außerordentlich rasch erfolgt, ist eine Abdichtung nicht in dem Maße notwendig, wie sie bei allen Formaldehydverfahren angebracht ist, die mit Apparaten arbeiten. Denn infolge der fast augenblicklich eintretenden Sättigung der Luft mit den Autandämpfen,

1) Siehe ihrell Aufsatz ln No. 6 (1907) dieser Wochenschrlit sowie in Zentralblatt fur Bukleriologic 1907, hier auch Literatur die durch hygrometrische Versuche von uns kontrolliert ist, erfolgt im allgemeinen eine viel raschere Niederschlagsbildung, als wenn erst innerhalb einer halben bis einer Stunde die Anhäufung der Dämpfe im Raume stattfindet, wie es bei Benutzung von Apparaten der Fall ist. Infolgedessen sind auch, selbst wenn nicht alle feinsten Ritzen eines Raumes sorgfialtig abgedichtet sind, die Gasverluste gering. Es ist aber damit nicht gesagt, die Abdichtung auch beim Autanverfahren im Prinzip nicht genau so durchzuführen, wie es von Flügge und seinen Schïlern empfohlen worden ist. Die Desinfektionseffekte sind bei Benutzung genügender Mengen von Autan zufriedenstellende. Del Einführung des Präparates ist allerdings der Preis hinderlich, der sich im Vergleich zu anderen Verfahren, z. B. dem Lingnerschen höher stellt. Nach den Berechnungen von Gastpar (Württemb. med. Corresp.-Blatt 1907) beträgt die Preisdifferenz zwischen Breslauer Methode und Autanverfahren $40 \%$. Aber diesem höheren Preis steht die Einfachheit der Ausführung, der Mangel der Feuelgefāhrlichkeit, der Wegfall der Anschaffungs-, Transport- und Reparaturkosten der Apparate und eine nicht unerhebliche Zeitersparnis, weil ja die Ueberwachung der Formaldehydentwicklung durch die Desinfektoren wegfällt, als Aequivalent gegenüber. Wenn man von der Einführung des Autanverfahrens sich oine Popularisierung der Wohnungsdesinfektion versprechen darf, so ist unter keinen Umständen damit gesagt, dab die Desinfektion den geschulten Desinfektoren und denjenigen, welche dieselbe amtlich und gesetzlich zu überwachen haben, entzogen werden soll. Davon kann schon deshalb keine Rede sein, weil ja die Formaldehyddesinfektion nur ein Teil der allgemeinen Wohnungsdesinfektion ist. Diese kann erfolgreich und in gröberem Unlfange nur durch geschulte Desinfektoren unter Aufsicht, bzw. Kontrolle von Aerzten oder deren Stellvertretern durchgeführt werden.

Seit der ersten Mitteilung Wesenbergs über seine mit Autan angestellten Versuche sind eine ganze Anzahl Arbeiten über den gleichen Gegenstand erschienen. Auf diejenigen $\mathrm{Pu}$ blikationen, welche mit den von mir skizzierten Ergebnissen im wesentlichen übereinstimmen, möchte ich hier nicht näher eingehen. Es sind hier die Arbeiten von Selter, von Nieter aus dem C. Fränkelschen Institute, von Xylander aus dem kaiserlichen Gesundheitsamte und diejenigen von Heller und Tomarkin aus dem Berner Institute zu erwähnen. Nieter, der unter C. Fränkel gearbeitet hat, verlangt auch für das Autanverfahren eine peinliche Abdichtung in jedem Falle. Es läßt sich gegen ein solches Verlangen kaum etwas einwenden, weil durch die Abdichtung die Desinfektionseffekte auf jeden Fall erhöht werden.

In einer Arbeit, deren experimenteller Teil im hygien ischen Institut der Univel'sität Berlin angefertigt ist, ubt Christian (Archiv f. Hygiene 1907) an den Arbeiten der genannten Autoren zum 'T'eil recht scharfe, aber, wie mir scheint, unberechtigte Kritik. Christian behauptet u. a., dals Bakterien, in Bouillon oder physiologischer Kochsalzlösung aufoeschwemmt, beim Eintrocknen stark geschädigt würden, und zwar durch den Sal\%gehalt dieser Substrate. Wi verlangt deshalb, die für Prüfung des Desinfektionseffektes bestimmten Testobjekte mit Bakterienkulturen herzustellen, welche in sterilem Brunnenwasser verdünnt sind. 'Es handeit sich hier um eine theore. tische Spekilation ohne experimentelle Grundlage. Der Einwand Christians hat für die Beurteilung der genannten Arbeiten keine Bedeutung, weil große Mengen von entwicklungsfahigen Bakterien an den Testobjekten auf Grund der Kontrollproben in jedem Falle nachgewiesen wurden. Ohristian behauptet ferner, daß bei seinen Versuchen die Autandämpfe eine geringere Fähigkeit entwickelt hätten, in die toten Winkel des Raumes einzudringen, als die auf andere Weise, z. B. mittels Apparaten erzeugten Formaldehyddämpfe. In den unter meiner Leitung ausgeführten Versuchen ist ein solches Verhalten der Autandumpfe nicht beobachtet worden. Es ist auch unwahrscheinlich, daß die Beobachteng von Christian verallgemeinert werden könnte, denn es ist kein physikalischer Grund yorhanden, weshalb sich die Autandämpfe anders als jedes gleichartige Gasgemenge verhalten sollten. Durch die bei der Versuchsanordnung Christians erhaltenen Resultate wird ferner der Anschein erweckt, als ob die Temperatur der Luft eines zil desinfizierenden Raumes von grolsem Einfluls auf den Ausfall der. Versuche wäre. Gerade in nnseren, bei den verschiedensten Temperaturen (von $\delta^{\circ}$ bis $26^{\circ} \mathrm{C}$ ) angestellten Versuchen zeigte es sich, daß die Temperatur der Luft des Raumes selb st von keinem 
Einflusse auf den Ausfall des Versuches ist und duls bei Benutzung genügend großer Autanmengen durch starke Niederschlagsbildung, \% B. an den Fenstern, an kühlen Flächen etc. die Desinfektionsffekte an den andern Flächen nicht herabgesetzt waren. Große Unterschiede der Temperatur der Luft und der im Raume enthaltenen Gegenstände werden die Desinfektionseffekte der Autandämpfe eher erhöhen als herabsetzen. Dagegen ist ohne weiteres aus physikalischen Grïlden verständlich, daß das Vorbandensein einzelner großer kühler Flächen, welche viel Autandämpfe mit dem Wasser an sich reißen, die Desinfektionseffekte an den wärmeren Flächen unter Umständen ungtinstig beeinflussen kann, nämlich dann, wenn nicht Autandämpfe im Ueberschuß vorhanden sind. In solchen Fällen wird es notwendig sein, die Menge des zu verdampfenden Autans zu vermehren, sodaß ein Leberschuß von Formaldehyd vorhanden ist.

Sehl wenig einwandfrei sind die Schlubfolgerungen, welche Chlistian aus seinen mit ganz kleinen Mengen Autan in Glaskolben angestellten Versuchen auf die Verhältnisse bei der Desinfektion großer Räume mittels des Autans zieht. Es erschien von vornherein zweifelhaft, daß eine derartige Schlubfolgerıng zulässig war. Es sind daher auf meine Veranlassung vorgleichende Versuche angestellt worden, um den Einflub der Menge des benutzten Autans, sowie die Entwicklung der Dämpfe in offenen und geschjossenen Gefäben, in guten und schlechten Wärmeleitern auf die Menge des entwickelten Gases und Wasserdampfes zu prüfen. Die Wassorverlustzahlen geben einen guten Indikator für die Menge der beim Autanprozeh entwickelten Wärme und daher auch für den Ablauf und die Intensität des chemischen Prozesses und erlauben deshalb einen Rückschluß auf die Menge des verdampften Formaldehyds. Der Sicherheit halber und um Vergloichswerte zu erhalten, wurde bei den mitzliteilenden Versuchen aber allch das Formaldehyd direkt bestimmt, und zwar nach Romijn (Zeitschrift für analytische Chemie 1897, Bd. 36, S. 18).

Es wurden $10 \mathrm{ccm}$ des Wassers, durch welches eine bestimmte Menge der mit Autandämpfen gesättigten Luft durchgesaugt war, mit $25 \mathrm{ccm}$ 1/10 Nornaljodlösung und $15 \mathrm{ccm}$ Normalnatronlauge fïnf Minuten lang stehen gelassen. Dann werden $20 \mathrm{ccm}$ Normalschwefelsaure hinzugefügt und schlieBlich der Ueberschuls des Jods mit 1/10 Normalthiosulfat (Stärke als Indikator) zurücktitriert. Es ist dabei zu beachten, daß stets ein genigender UebelschuB der Jodlosung benutzt wird. Es müssen mindestens $10 \mathrm{ccm}$ Thiosulfatlösung erforderlich sein. Andernfalls ist der Versuch mit nur $5 \mathrm{ccm}$ Formaldehydlösung zu wiederholen, da sonst die Bestimmung nicht genau ausfällt. $1 \mathrm{ccm}$ der von der Flüssigkeit verbrauchten Jodlösung entspricht $1,5 \mathrm{mg}$ Formaldehyd $\left(\mathrm{CH}_{\mathbf{2}} \mathrm{O}\right)$.

Dieses Verfahren dürfte, wenn auch etwas komplizierter, so doch exakter als das von Christian, nach Seyewitz und Gibello, benutzte sein. Denn bei letzterem ist der Farbenumschlag des Phenolphthaleins nicht präzis genug, um genaue Bestimmungen alszuführen. Es seien folgende Zahlen wiedergegeben:

1. Versuch. $75 \mathrm{~g}$ Autan, Gewichtsverlust $28 \mathrm{~g}=$ Formaldehyd 2,93 $\mathrm{g}$; auf $1 \mathrm{~kg}$ berechnet $370 \mathrm{~g}$, Gewichtsverlust $=39,0 \mathrm{~g}$ Formaldehyd.

2. Versuch. $100 \mathrm{~g}$ Autan, $35 \mathrm{~g}$ Gewichtsverlust $=3,27 \mathrm{gr}$ Formaldehyd, auf $1 \mathrm{~kg}$ berechnet $350 \mathrm{~g}$ Gewichtsverlust $=32,7 \mathrm{~g}$ Formaldehyd.

3. Versuch. $100 \mathrm{~g}$ Aatan, $42 \mathrm{~g}$ Gewichtsverlust $=-3,78 \mathrm{~g}$ Formaldehyd, auf $1 \mathrm{~kg}$ berechnet $429 \mathrm{~g}$ Gewichtsverlust $=37,8 \mathrm{~g}$ Formaldehyd.

Es handelt sich bei allen diesen Versuchen, von denen einige Beispiele mitgeteilt sind, um Experimente mit verschiedenen Proben des Autans. Wie wesentlich gerade bei den kleineren Versuclismengen die Beobachtung von Einzelheiten ist, goht unter anderm aus del nachstehenden, kleinen Tabelle hervor, welche den Einfluß der bei der Reaktion zu erwärmenden Glasmenge zeigt. Je größer dieselbe ist, desto geringer die Wärmeentwicklung und daher auch die Menge des verdampiten Wassers und des entwickelten Formaldehyds. Zur Reaktion kommen von demselben Präparat je $75 \mathrm{~g}$.

\begin{tabular}{c|c|c}
\hline $\begin{array}{c}\text { Gewicht des Glas- } \\
\text { kolbens }\end{array}$ & Gewichtsverlust & $\begin{array}{c}\text { Formaldehyd- } \\
\text { menge }\end{array}$ \\
$=-0$ & & \\
$200 \mathrm{~g}$ & $30 \mathrm{~g}$ & $2,88 \mathrm{~g}$ \\
$350 \mathrm{~g}$ & $29 \mathrm{~g}$ & $2,66 \mathrm{~g}$ \\
$466 \mathrm{~g}$ & $27 \mathrm{~g}$ & $2,25 \mathrm{~g}$
\end{tabular}

Daß die Wasserverlustzahlen, welche Christian bei seinen Versuchen mit kleinen Mengen Autan gefunden hat, viel zu gering sind, davon kann man sich jeden Augenblick durch die Anstellung eines Versuches mit größeren Mengen des Autans, von 1000 bis $2000 \mathrm{~g}$, überzeugen. Wie aus größeren Versuchsreihen hervorgeht, betragen die Gewichtsverluste bei Verwendung von $1 \mathrm{~kg}$ Autan und $900 \mathrm{ccm}$ Wasser im allge. meinen zwischen 450 und $500 \mathrm{~g}$. Aus folgender Tabelle kann der Einflub, welchen die Benutzung verschiedenartiger Gefäße auf die Entwicklung des Autans bei kleinen Mengen ausübt, leicht ersehen werden.

\begin{tabular}{|c|c|c|c|}
\hline & Porzellanschal & $\begin{array}{l}\text { asser we } \\
\text { Wasser } 23^{\circ}\end{array}$ & Verlus \\
\hline 1. & 20,0 & 18,0 & 9,5 \\
\hline 2. & 40,0 & 36,0 & 19.0 \\
\hline 3. & 100,0 & 90,0 & 43,0 \\
\hline \multicolumn{4}{|c|}{ Orfener Kolben; $10 \%$ Wasser weniger. } \\
\hline 4. & 20,0 & 18,0 & 3,5 \\
\hline 5. & 50,0 & 36,0 & 8,0 \\
\hline 6. & 100,0 & 90,0 & 35,0 \\
\hline \multicolumn{4}{|c|}{ Kolben mit aufgesetztem Rolur. } \\
\hline$y$ & $\$ 0,0$ & 36,0 & 8,0 \\
\hline 8. & 100,0 & 90,0 & 29,0 \\
\hline \multicolumn{4}{|c|}{ Offene, lobe Blechbichse. } \\
\hline 9. & 20,0 & 18,0 & 8,0 \\
\hline 10. & 40,0 & 36,0 & 16,0 \\
\hline 11. & 100,0 & 90,0 & 43,0 \\
\hline \multicolumn{4}{|c|}{ Porzellatuschale, ticht umgeruhrt. } \\
\hline 12 & 20,0 & 18,0 & 8,0 \\
\hline 13 & 40,0 & 36,0 & 16,5 \\
\hline 14. & 100,0 & 90,0 & 42,0 \\
\hline
\end{tabular}

Es geht aus diesen Versuchen hervor, daß bei Kntwicklung der Autandämpfe in einem offenen Gefäg eine viel stärkere Reaktion und damit eine stärkere Verdampfung des Wassers erfolgt als bei Anwendung eines Kolbens.

Aus diesen Angaben dürfte schon zur Genüge klar werden, auf wie schwachen Füßen die Christiansche Kritik der Arbeiten von Selter, Nieter, Wesenberg, Heller und Tomarkin steht. Christian hat die beiden Fundamentalfehler gemacht, aus Versuchen mit kleinen Mengen auf die Verhältnisse im großen Schlïsse und bei den Versuchen im kleinen selbst nicht in Betracht gezogen zu haben, inwieweit die Versuchsanordnung das Resultat beeinfluft.

Dazu kommt, daf Christians Zahlen bei gleicher Versuchsanordnung so große Verschiedenheiten aufweisen, daß entweder Fehler bei den Versuchen vorgekommen sein müssen, oder daß die Untersuchungsmethode nicht einwandsfrei ist. Seine Versuche 3, 8, 10 und 12 sind in gleicher Weise angestellt und ergeben trotzdem Unterschiede von 20,10 und $40 \%$ untereinander. Versuch 1 mit $10 \mathrm{~g}$ ergab z. B. 16,5 g Formaldehyd auf das Kilo, Versuch 12 mit $20 \mathrm{~g} 17,5$.

Trotzdem das Autanverfahren keineswegs als ein Idealverfahren hingestellt werden kann, ist es doch mit Freude als der erste Schritt auf einem Wege zu bezeichnen, der aus sehr praktischen Gesichtspunkten gewählt ist. Zur Popularisierung des Verfahrens ist es notwendig, zunächst den Preis des Präparates herabzusetzen bei gleichzeitiger Erhöhung der bisher von der Fabrik angegebenen Menge. Oder es muß versucht werden, die Desinfektionseffekte zu erhöhen, vielleicht dedurch, daß man die Bestandteile des Autans (Paraform und Metallsuperoxyd) in getrennten Päckchen aufbewahrt und erst dann mischt, wenn die Desinfektion vorgenommen werden soll Vielleicht lassen sich auch durch andere Zusammensetzung des Präparates noch bessere Desinfektionseffekte erzielen. Vor allem aber müssen bestimmte Vorschriften für die Einleitung der Reaktion gegeben und von den Desinfektoren stets beachtet werden. Am geeignetsten verläuft die Reaktion bei Zusatz von Wasser, welches die Temperatur von $20-25^{\circ} \mathrm{C}$ hat. Als Behälter müssen solche aus schlechten Wärmeleitern hergestellte dienen, vor allen Dingen Holzbottiche und Waschzuber. Damit die Reaktion gut verläuft, muß ein sorgfältiges Verrühren des Pulvers mit dem Wasser erfolgen. Wird zu heißes Wasser benutzt, so verläuft die Reaktion so rasch, daß ein sorgfältiges Umrühren nicht möglich ist. Es wird infolgedessen nicht die ganze Autanmenge in Reaktion treten. 
Wenn ich von einer Popularisierung der Wohnungsdesinfektion gesprochen habe, so möchte ich dem Mißverständnis vorbeugen, als ob durch Einführung des Autanverfahrens die geprüften Desinfektoren ausgeschaltet werden sollten. Dieser irrtümlichen Auffassung ist Kirstein verfallen und hat deshalb gegen die Einführung des Autans Stellung genommen. Es würde auch verkehrt sein, wenn man die Wohnungsdesinfektion in zu großem Umfange anwenden und im Vertrauen auf die Schlußdesinfektion die Unschädlichmachung der Ex- und Sekrete der Kranken während der Dauer der Krankheit vernachlässigen wollte. Es wird ferner durch das Autanverfahren keineswegs das Verlangen nach einem noch wirksameren gasförmigen Desinfektionsmittel beiseite gedrängt werden, nach einem Mittel, welches nicht nur die Oberflächen noch stärker desinfiziert, sondern auch in die Tiefe der Gegenstände eindringt und zu gleicher Zeit das Ungeziefer vernichtet. Erst durch ein solches Mittel würde, wenn es ebenso wenig schädliche Nebenwirkungen wie das Formaldehyd hat, die Frage der Wohnungsdesinfektion ihrer endgültigen Lösung näher gebracht sein.

\section{II.}

Für die Händedesinfektion, deren Prinzipien ich kurz kritisch beleuchten möchte, sind zahlreiche Verfahren angegeben, von denen, wenigstens in Deutschland, die bekanntesten und am meisten gebrauchten die Fürbringersche Methode der Desinfektion mit Seife, Alkohol und Sublimat, sowie die Ahlfeldsche Methodeder Desinfektion mit Seife und Alkohol geworden sind. Aber auch die Karbolsäure sowie das Lysol und Sublimat allein werden von vielen Aerzten und Chirurgen zur Reinigung bzw. Desinfektion der Hände benutzt. In den letzten Jahren ist zu diesen Methoden noch die Anwendung des Seifenspiritus, die von Mikulicz und Vollbrecht empfohlen wurde, hinzugekommen, und ganz neuerdings hat Schumburg ein Verfahren angegeben, auf das ich noch zu sprechen komme und dessen Vorzüge mir seine Anwendung, namentlich für die Zwecke des Krieges, angebracht erscheinen lassen. Bei allen diesen Verfahren wird eine sorgfältige mechanische Reinigung der Hand mit Bürste und Seife, eventuell mit Marmorseife, vorausgesetzt. Aber jeder Chirurg oder Gynäkologe weif, daß alles Desinfizieren unter Umständen im Stich läßt, namentlich dann, wenn es sich darum handelt, die mit virulenten Infektionserregern beschmutzten Hände von diesen wieder zu befreien. Man kann sogar behaupten: Das Problem einer wirklichen Desinfektion der Hände ist noch ungelöst. Wir verfügen bisher noch über kein Verfahren, die Haut von Infektionsstoffen, welche sich in der Tiefe der Epidermis, namentlich der Drüsen und Haarbälge befinden, völlig zu befreien. Man kann von einer eigentlichen Desinfektion der Hände deshalb überhaupt nicht sprechen. Das läßt sich leicht durch die bakteriologische Kontrolle des Keimgehaltes der Haut beweisen. Durch die bakteriologischen Untersuchungen können zwei Tatsachen von grundlegender Bedeutung festgestellt werden: nämlich erstens, daß es nicht gelingt, eine Hand in toto völlig keimfrei zu machen, und zweitens, daß durch Maßnahmen, welche die oberen Schichten der Epidermis auflockern, auch bei den an. scheinend keimfreien oder wenigstens keimarmen Händen eine gewaltige Ablösung der in den tiefen Schichten enthaltenen Hautkeime erfolgt. Diese Zunahme der Keime, wie sie auch bei dem sorgfältigsten Desinfizieren der Hände nach reichlicher Anwendung von steriler Seife erfolgt, ist eine um so größere, je mehr die Hände mit Haaren bedeckt sind, je größer die Zahl der Drüsen an ihrer Oberfläche ist und je mehr rallhe oder gar rissige Stellen sich auf der Haut finden. Denn gerade die kleinen und kleinsten Schrunden sind der Sitz von zahllosen Mikroorganismen, darunter gerade häufig von pathogenen Bakterien. Schumburg hat in seiner kriegschirurgisch wichtigen Arbeit diese Tatsache durch sorgfältige, vielfach modifizierte Versuche bewiesen, und die im Berner Institut unter meiner Leitung von Tomarkin und $\mathrm{Heck}$ angestellten Versuche haben das gleiche dargetan. Die Hände werden in den tieferen Schichten der Haut, wie sich wiederum durch anhaltendes Seifen nachweisen läßt, um so keimarmer, je mehr sie gepflegt werden und je geringer die Zahl der rauhen und mit stärkeren Haaren besetzten Stellen ist. Die Chirurgen haben mit Recht die Forderung aufgestellt, dab es darauf ankommt,

1. die Hand vor der Infektion mit gefährlichen Infektionserregern möglichst zu schützen;

2. die Hand, wenn die Annahme einer Infektion besteht oder möglich ist, keimdicht vom Operationsfelde zu trennen.

Auf Grund der ersten Folgerung ist Kocher logischer. weise zu der Forderung gelangt, daß man die Handschuhe dann tragen soll, wenn man infektiöse Kranke untersucht oder behandelt, und daß man die Handschuhe zu den Operationen ausziehen soll. Es ist ganz sicher, daß diese von Kocher geforderte Prophylaxis einer Infektion der Hände eine außerordentlich wertvolle Maßnahme in der Verhütung der Wundinfektion bildet.

Um der zweiten Forderung zu genügen, sind von Mikulicz und seiner Schule die über Gummihandschuhe zu tragenden dünnen Baumwollhandschuhe in die chirurgische Praxis eingeführt. Aber diese Handschuhe haben mancherlei Nachteile und werden, weil namentlich das Gefühl der Hand so außerordentlich beschränkt wird, von manchen Autoren verworfen. Man hat versucht, sie zu ersetzen dadurch, daß die Hand mit einem undurchlässigen Ueberzug überzogen wird, welcher ein Uebertreten der Keime aus der Haut in die Flüssigkeiten des Operationsgebietes verhütet. Die Hand wird also mit Lösungen bepinselt, welche erstarren und so die Haut mit ihren Anhängen (Drüsen) hermetisch abschließen. Diese Anstriche der Hand können zugleich desinfizierende Zusätze enthalten. Eine Bedingung für ihre Anwendung ist, daß sie nicht reizend auf die Hand wirken, daß sie sich leicht entfernen lassen und daf sie sich in den Gewebsflüssigkeiten nicht lösen. Auch müssen sie elastisch sein. Ein sehr empfehlenswerter derartiger Handanstrich scheint das ganz neuerdings von Klapp und Dönitz empfohlene Chirosotër zu sein, eine wachsartige Masse, der flüssige Harze zugesetzt sind. Es liegt hier sicher ein Gebiet vor, auf welchem die Technik berufen ist, noch weitere Fortschritte zu erzielen. Denn solche Ueberzüge lassen sich nicht nur mit Antisepticis imprägnieren, sondern sie können auch (wie z. B. das Chirosotēer) zur Konservierung der Hautoberfläche dienen, indem sie die Haut geschmeidig erhalten.

Der Kernpunkt der Händedesinfektion für die Praktiker mub aber, selbst wenn ein solcher idealer Handanstrich gefunden werden sollte, ein einfaches, sicheres und die Hände nicht angreifendes Verfahren, bei welchem die Haut mit iöslichen Mitteln möglichst keimfrei gemacht wird, sein. Als ein solches Verfahren möchte ich das von Schumburg empfohlene hinstellen, über das ich von den Herren Tomarkin, Heck und Dahinden ausgedehnte Versuchsreihen habe anstellen lassen. Schumburg verwendet zur Desinfektion der Haut eine Mischung von Alkohol und Aether $(2: 1)$, welcher $0,5 \%$ Salpetersäure zugesetzt ist. Mit dem Alkoholäthersäuregemisch wird die Haut mit Hilfe von Wattebäuschchen intensiv abgerieben. Das Gemisch wirkt namentlich vermöge seines Säuregehaltes nicht nur desinfizierend, sondern führt auch eine Schrumpfung der Hautoberflächen herbei, wodurch die Keime fixiert werden; selbst bei langdauernden Operationen findet eine Aufweichung der tieferen Schichten der Haut, welche in dieser Weise vorbehandelt ist, nicht statt. Die Waschflüssigkeit oder die Gewebsflüssigkeiten, in welchen die Hand operiert, bleiben, wie Heck unter meiner Leitung bei zahlreichen von Prof. Tavel ausgeführten Operationen durch viele Versuche feststellen konnte, auBerordentlich keimarm. Wegen der Einfachheit des Verfahrens ist dasselbe für die Kriegschirurgie demnach von großer Bedeutung, um so mehr, als es, wie unsere eigenen Versuche, sowie die Beobachtungen von Prof. Tavel, der seit langer Zeit vor allen Operationen seine Hände in Schumburgs Lösung wäscht, zeigen, außerordentlich schonend ist. Die Haut wird in keiner Weise angegriffen und erhält sich ohne Risse und Schrunden, ja bei dauernder Anwendung werden Hände, an denen sich durch Benutzung der gewöhnlichen Desinfektionsmittel ekzematöse oder rissige Stellen gebildet haben, bald völlig glatt und weich.

Ich glaube, durch meine Ausführungen hinreichend bewiesen zu haben, dah in der Desinfektionsfrage zwar viele Fortschritte dank intensiver Arbeil der Hygieniker, Bakterio- 
logen und Praktiker erzielt sind, daß aber nicht minder zahlreiche Probleme der Lösung noch harren.

Li teratur: F1ügge, Zeitschrift für Hygiene 1898 , Bd. 29. - Schumburg Archiv für klinische Chirurgie Bd 79, H. I - A. E I c he ng $\mathrm{r}$ ä $\mathrm{n}$, Ein neues Formaldehyddesinfektionsverfahren, das Autanverfahren. Zeitschrift für angewandte Chemie $1906, \mathrm{H} 33$. - S elter, Bakteriologische Untersuchungen über ein neues Desinfektonsverfahren, das Autan. Münchener medizinische Wochenschrift 1906, No. 50 und Zeitschrilt fiur Medizinalbeamte 1906 , No. 24 . - We s e n ber $\mathrm{g}$. Die Formaldehyddesinfektion mit Autan. Hygienische Rundschau 1906, No. 22. - A. N i e t e r, Ueber die Formaldehyddesinfektion mit Autan. Hygienische Rundschau 1907, No. 3 Kirstein, Ueber eln neues Formaldehydpräparat „Autan“ zur Raumdesinfektion. Kirstein, eber eln neues Formaldehydpraparat "Autan zur Raumdesinfektion. Zeitschrift fur Medizinalbeamte 1907, No. 2. - Kolle, Ueber Wohnungs im besonder nenere Formaldehyd- sowie das Autanvent Pr. C. Franke,

Wochenschrift 1899. - Re ichen bach, Zeitschrift für Hygiene Bd. 50. - R u b ne nd P e e r e ntoom, Hygienische Rundschau 1899 ing Ueber die Formaldehyddesinfektion mit 1907, No. 6 und Zentralblatt fiir Bakteriol Aigie Bd 43 , und Experimentelles zur Autandesinfektlo $X \mathrm{y} l$ a n $\mathrm{r}$. Veruche

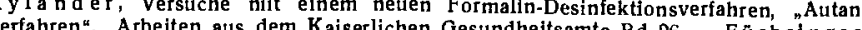
Intersuchungen und Wiesu To 2 18, 185, 3 , 1809, No. 2 und 48, 1895, No. 3; 1899 , No. 49. - A hif eld, Deutsche medizinische Wochen schrift 1895, No. 24; 1896 . No. 23; 1897, No. 8; Monatsschrift fut Geburtshilfe und Gynàkologie 1899 , Bd. 10, H. $1 / 2$; Zeitschrift für Medizinalbeamte 1898, No. 17/18. Mikulicz, Detutsche medizinische Wochenschrift 1899 , No. 24 und Deutsche militar arztliche Zeitschrift 1900, No. l. - Paul und Krönig, Zeitschrift far Hygiene 1897, d. 25. - Pfute, Deutsche militärărztliche Zeitschrift 1899 